\title{
Mechanism of the Vasodilator Action of Pinacidil
}

\author{
M.J. Mulvany, L.M. Videbaek, A.D. Hughes, C. Aalkjaer \\ Danish Biomembrane Research Center and Department of Pharmacology, Aarhus University, \\ Aarhus, Denmark \\ Corresponding author: Dr. M.J. Mulvany, Department of Pharmacology University of Aarhus \\ Universitetsparken 240, DK-8000 Aarhus C (Denmark)
}

\begin{abstract}
The mechanism of the vasodilator action of pinacidil has been studied in rat mesenteric small arteries. The results show, first, that the use of flux studies to make measurements of ion permeability requires knowledge of the membrane potential, especially as regards $\mathrm{K}+$ permeability. Second, the results confirm that the vasodilator effect of pinacidil is due to an increase in $\mathrm{K}^{+}$permeability. Lastly, the results suggest that the $\mathrm{K}+$ channels involved are sensitive to glibenclamide.
\end{abstract}

Key Words. Resistance vessels $\cdot \mathrm{K}+$ channels $\cdot$ Membrane potential $\cdot$ Ion permeability

\section{Introduction}

Pinacidil - like cromakalim, nicorandil and minoxidil - belongs to the class of vasodilators now known as $\mathrm{K}$ channel openers [1]. The hypothesis is that these drugs act by changing $\mathrm{K}+$ permeability, and thus the permeability of the calcium channels through direct control of the membrane potential. It is, however, only recently that direct evidence has been obtained supporting the concept that the drugs are activators of $\mathrm{K}$ channels [2-6]. The aim of this communication is 2-fold: (1) to describe our contribution [5, 7] to the growing evidence that these drugs do indeed cause vasodilatation by causing opening of $\mathrm{K}+$ channels, at least as regards pinacidil, and (2) to discuss new evidence concerning the type of channel which may be involved.

\section{Methods}

The methodology is described in detail elsewhere [5, 7]. In brief, rat mesenteric small arteries (i.d. 100-200 $\mu \mathrm{m}$ ) were mounted in a myograph [8] and suspended in a chamber containing oxygenated physiological salt bicarbonate-buffered solution at $37^{\circ} \mathrm{C}$. 
This allowed the isometric response of the vessels to the various drugs to be determined while simultaneous measurements of membrane potential were made using intracellular electrodes. Efflux rate constants were measured using the techniques developed for measurement of sodium metabolism in these vessels [9].

\section{Results and Discussion}

\section{Mechanism of Action}

We have previously shown that pinacidil causes a concentration-dependent relaxation of rat mesenteric small arteries, the $\mathrm{IC}_{50}$ being somewhat less than $1 \mu \mathrm{M}$, with full relaxation being obtained with $10 \mu \mathrm{M}$ [5]. Furthermore, we have shown that the effect of pinacidil is eliminated under conditions where the membrane is held depolarized in high potassium solution. This therefore raised the possibility that pinacidil acts by hyperpolarizing the cell membrane, and indeed this is the case: in resting vessels, starting from a resting potential of about $-60 \mathrm{mV}$, pinacidil causes a prompt concentration-dependent reversible hyperpolarization with an $\mathrm{EC}_{50}$ of about 1. and a maximum hyperpolarization of about $20 \mathrm{mV}$ with $10 \mu \mathrm{M}$ pinacidil [5]. This also is the case for activated vessels, where relaxation and hyperpolarization occur simultaneously (fig. 1). Since in rat mesenteric small arteries the membrane potential plays a dominant role in the determination of tone [10], there can be little doubt that the relaxation is primarily due to the hyperpolarization, but what is the mechanism by which pinacidil causes the hyperpolarization?

As described by the Goldman [11] equation, the membrane potential of a cell is determined by the concentrations of ions in- side and outside the cell, and the permeabilities of the cell membrane to these ions:

$$
E_{m}=-(R \cdot T / F) \cdot \ln \left[\frac{[K]_{i} \cdot P_{K}+[\mathrm{Na}]_{i} \cdot P_{\mathrm{Na}}+[\mathrm{Cl}]_{0} \cdot P_{\mathrm{Cl}}}{[\mathrm{K}]_{0} \cdot \mathrm{P}_{\mathrm{K}}+[\mathrm{Na}]_{0} \cdot \mathrm{P}_{\mathrm{Na}}+[\mathrm{Cl}]_{\mathrm{i}} \cdot \mathrm{P}_{\mathrm{Cl}}}\right]
$$

where $\mathrm{i}$ and o refer to the intracellular and extracellular spaces, respectively; P denotes permeability to the ions indicated; and $\mathrm{R}, \mathrm{T}$ and $\mathrm{F}$ have the usual meaning [12]. Thus hyperpolarization could be caused either by an increase in the $\mathrm{K}+$ permeability, or by a decrease in the $\mathrm{Na}^{+}$permeability, both of which would cause the cell to approach the equilibrium potential for $\mathrm{K}^{+}$. Changes in CI- permeability could also be involved, depending on whether the membrane potential is positive or negative with respect to the $\mathrm{Cl}^{-}$equilibrium potential. We have therefore investigated the effect of pinacidil on $\mathrm{K}^{+}, \mathrm{Na}^{+}$and $\mathrm{Cl}^{-}$permeability.

In resting vessels, pinacidil causes a concentration-dependent increase in ${ }^{42} \mathrm{~K}$ efflux, with an $\mathrm{EC}_{50}$ of about $1 \mu \mathrm{M}$, as for the hyperpolarizing effect of the drug. However, when these experiments were repeated in activated vessels the results were surprising (fig. 2.). It was found that when the washout solutions contained noradrenaline, pinacidil had no effect. 
The solution to this paradox lies in the realization that the efflux rate constant is dependent not only on the permeability but also on the membrane potential. Since pinacidil causes such a profound hyperpolarization, exposure to pinacidil will increase the potential barrier to $\mathrm{K}^{+}$: if the permeability to $\mathrm{K}^{+}$remained unchanged, we would expect the efflux to decrease. Thus the fact that the efflux does not change can only be explained in terms of the $\mathrm{K}$ permeability increasing. The permeability can be calculated [7] from the so-called Katz factor [13], a factor which depends on the membrane potential:

efflux rate constant $=$ constant $\cdot \mathrm{P} \cdot($ Katz factor $)$

For a cation like $\mathrm{K}^{+}$, and a negative membrane potential, the Katz factor is less than unity indicating that the efflux is less than would otherwise be expected, since the $\mathrm{K}^{+}$has to efflux against this potential barrier. Calculation of the permeability requires account to be taken of both the efflux rate constant and the membrane potential.

On this basis we have combined the measurements made of membrane potential and efflux rate constant to make statistical estimates of the permeability changes. These calculations show that there is indeed an increase in $\mathrm{K}^{+}$permeability (fig. 3 ), both in resting and in activated vessels. Thus it appears that pinacidil is indeed a drug which acts primarily by increasing $\mathrm{K}^{+}$ permeability.

\section{Channel Characterization}

We may now consider the type of channel which may be involved. As shown above, investigations of this type are complicated by the requirement for measurements of both efflux rate constant and membrane potential. To some extent the difficulties may be overcome (as suggested to us by Dr. A.P. Somlyo) if the efflux experiments are performed in a high $\mathrm{K}^{+}$ solution, for then the membrane potential would be about zero and independent of opening of $\mathrm{K}^{+}$ channels.

As in normal saline, pinacidil also causes an increase in $\mathrm{Rb}^{+}$(here used as a marker for $\mathrm{K}^{+}$) efflux (fig. 4a) in high $\mathrm{K}^{+}$solution. In the presence of D600, the Rb efflux rate constant was markedly lower (presumably because the open probability of calcium-dependent $\mathrm{K}^{+}$channels was reduced). However, the ability of pinacidil to enhance $\mathrm{K}$ efflux was not inhibited by 0600 (fig. 4b), suggesting that the channels concerned are not sensitive to calcium. This provides some evidence against the $\mathrm{K}$ channels concerned being calcium-activated $\mathrm{K}^{+}$channels (in agreement with Winquist et al. [14]; but see Hermsmeyer [3]). The effect of pinacidil was, however, inhibited by the sulphanylurea, glibenclamide (fig. 4c), a drug which can inhibit ATP-dependent $\mathrm{K}^{+}$channels [15). Furthermore, we have found that glibenclamide (1 and $\left.10 \mu \mathrm{M}\right)$ completely inhibits the ability of pinacidil to relax these vessels [data not shown], confirming data from 
other vessels $[2,13)$. The data therefore sup- port the possibility that the $\mathrm{K}$ channels involved are ATP-sensitive, but more work is necessary to establish this.

\section{Acknowledgements}

This work has been supported by the Danish Medical Research Council and the Leo Pharmaceutical Company.

\section{References}

1 Cook NS: The pharmacology of potassium channels and their therapeutic potential. Trends Pharmacol Sci 1988;9:21-28.

2 Standen NB, Quayle J, Davies NW, et al: Hyperpolarizing vasodilators activate ATP-sensitive K channels in arterial smooth muscle. Science 1989; 245:177-180.

3 Hermsmeyer K: Ion channel effects of pinacidil in vascular muscle. Drugs 1988;36(suppl 7):29-32.

4 Southerton JS, Weston AH, Bray K.M, et al: The potassium channel opening action of pinacidil; studies using biochemical, ion flux and microelectrode techniques. Naunyn Schmiedebergs Arch Pharmacol 1988;338:310-318.

5 Videbaek LM, Aalkjaer C, Mulvany MJ: Pinacidil opens $\mathrm{K}^{+}$-selective channels causing hyperpolari- zation and relaxation of noradrenaline contractions in rat mesenteric resistance vessels. Br J Pharmacol I 988;95:103-108.

6 Quast U, Baumlin Y: Comparison of the effluxes of ${ }^{42} \mathrm{~K}^{+}$and ${ }^{86} \mathrm{Rb}^{+}$elicited by cromakalim (BRL 34915) in tonic and phasic vascular tissue. Naunyn Schmiedebergs Arch Pharmacol 1988;338:319-326.

7 Videbaek LM, Aalkjaer C, Hughes AD, Mulvany MJ: Effect of pinacidil on ion permeability in rest- ing and norepinephrine contracted rat resistance vessels. Am J Physiol, in press.

8 Mulvany MJ, Halpern W: Contractile properties of small arterial resistance vessels in spontaneously hypertensive rats. Circ Res 1977;41:19-26.

9 Aalkjaer C, Mulvany MJ: Sodium metabolism in rat resistance vessels. J Physiol 1983;343:105-116.

10 Mulvany MJ, Nilsson H, Aatman JA: Role of membrane potential in the response of rat small mesenteric arteries to exogenous noradrenaline stimulation. J Physiol 1982;332:363-373.

11 Goldman DE: Potential, impedance, and rectification in membranes. J Gen Physiol 1943;27:37-60. 
12 Jones AW: Content and fluxes of electrolytes; in Bohr DF, Somlyo AP, Harvey HV (eds): Vascular Smooth Muscle. Handbook of Physiology, Section 2, vol 2. Bethesda, American Physiological Society, 1980, pp 253-299.

13 Hodgkin AL, Katz B: The effect of sodium ions on the electrical activity of the giant axon of the squid. J Physiol 1949;108:37-77.

14 Winquist RJ, Heaney LA, Wallace A, et al: Glyburide blocks the relaxation response to BRL 34915 (cromakalim), minoxidil sulphate and diazoxide in vascular smooth muscle. J Pharmacal Exp Ther 1989;248:149-156.

15 Arena JP, KassA: Enhancement of ATP-sensitive K-channel activity in heart cells by pinacidil. Biophys J 1989;55:585a. 


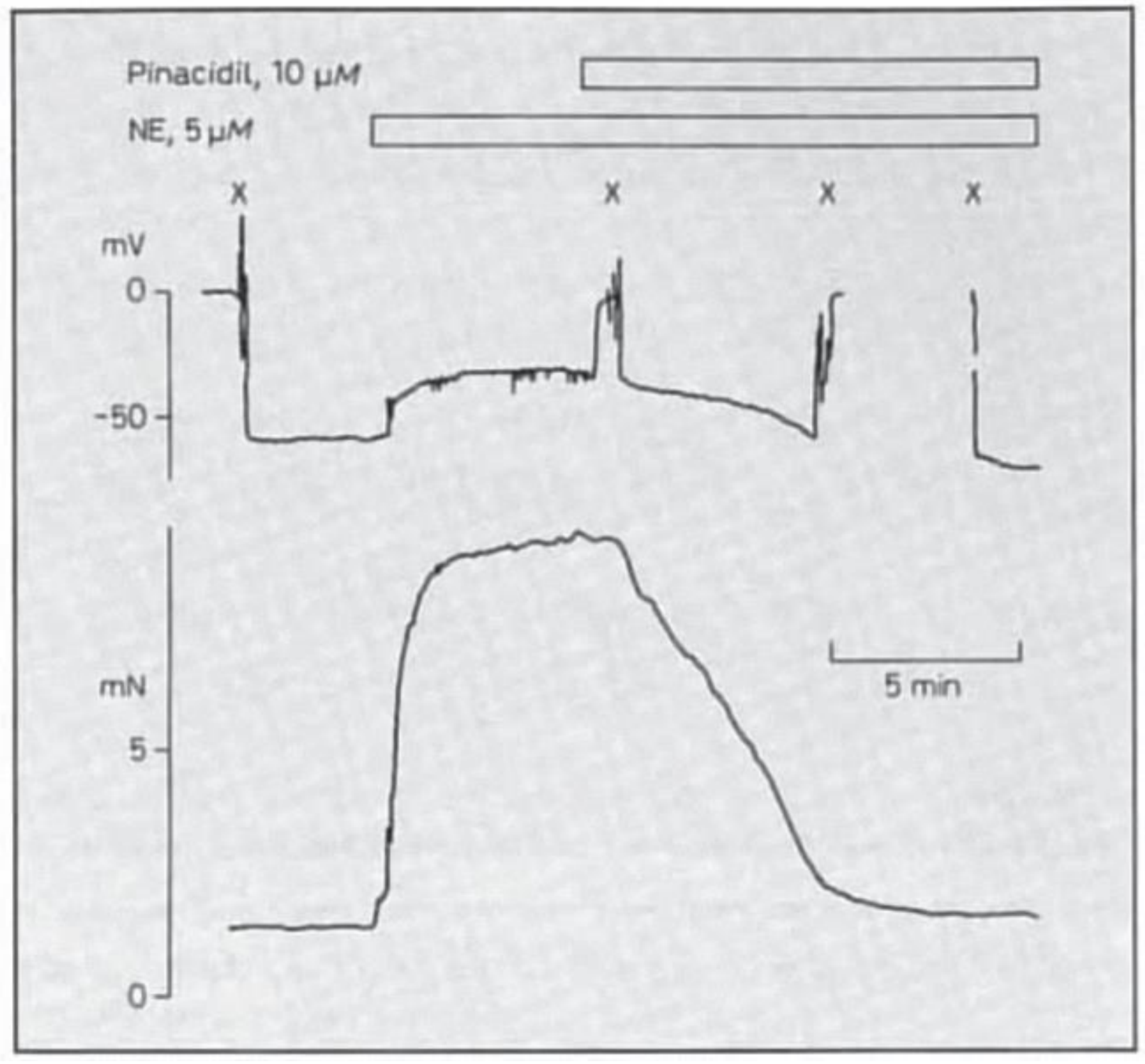

Fig.l. Simultaneous measurements of the effect of pinacidil on tone and membrane potential of an activated rat mesenteric small artery. Electrode withdrawn at each $\mathrm{X}$ to show extracellular potential. Unpublished record from experiments reported in Videbaek et al. [5]. 


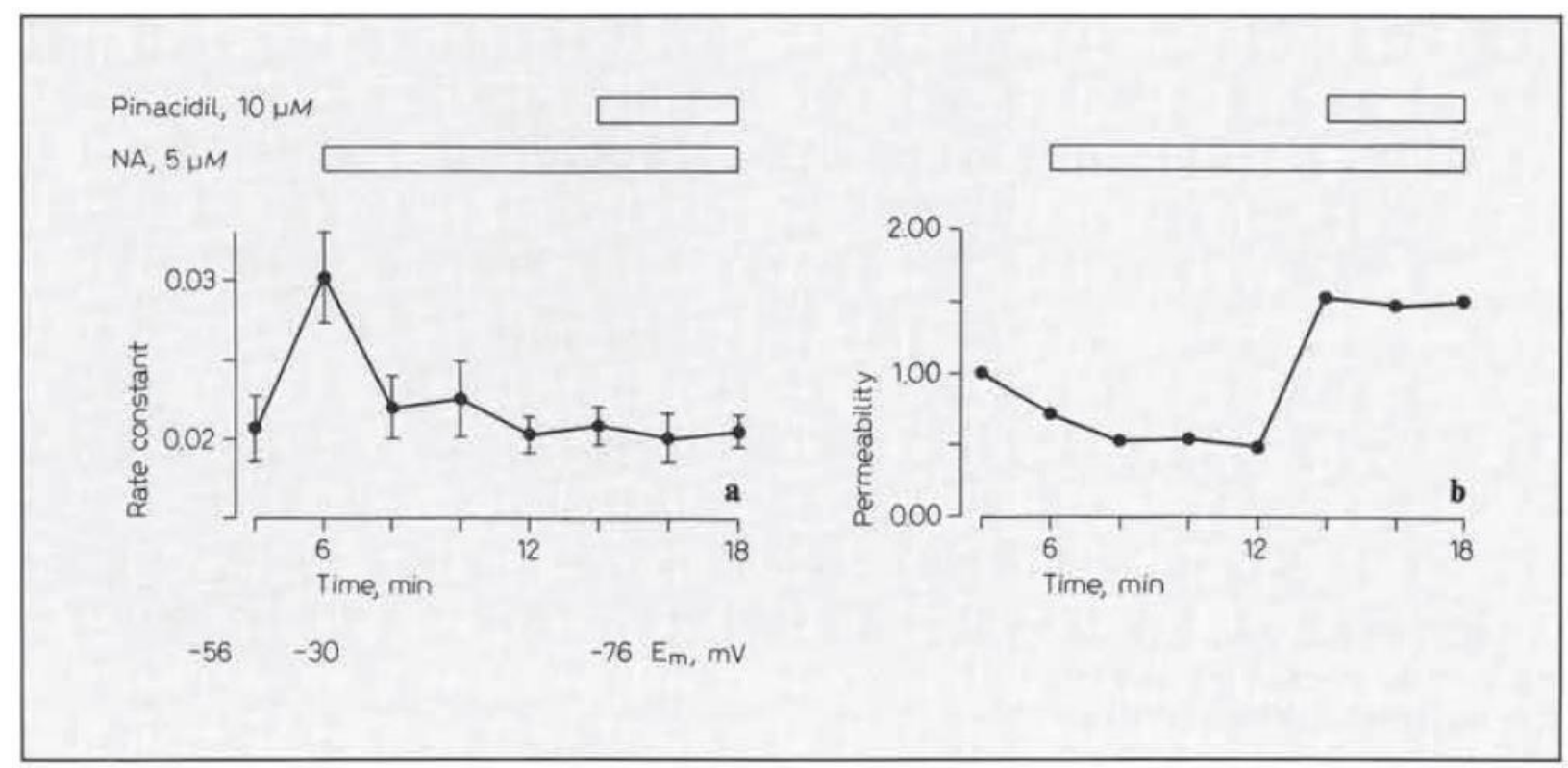

Fig.2. $\mathbf{a}^{42} \mathrm{~K}$ efflux rate constant (mean \pm SEM) from rat mesenteric small arteries. Vessels loaded with ${ }^{42} \mathrm{~K}$ as described in Videbaek al. [7] and washed out in saline solutions $\left(37^{\circ} \mathrm{C}, \mathrm{pH}\right.$ $7.4,\left[\mathrm{Ca}^{2+}\right] 2.5 \mathrm{mM}$ ) containing noradrenaline (NA) and pinacidil as shown. Abscissa shows times after start of washout. Numbers beneath show values of membrane potential, $\mathrm{E}_{\mathrm{m}}$, measured on a myograph [7), but otherwise under similar conditions. b Corresponding mean permeability values (arbitrary units) calculated as de- scribed in the text. 


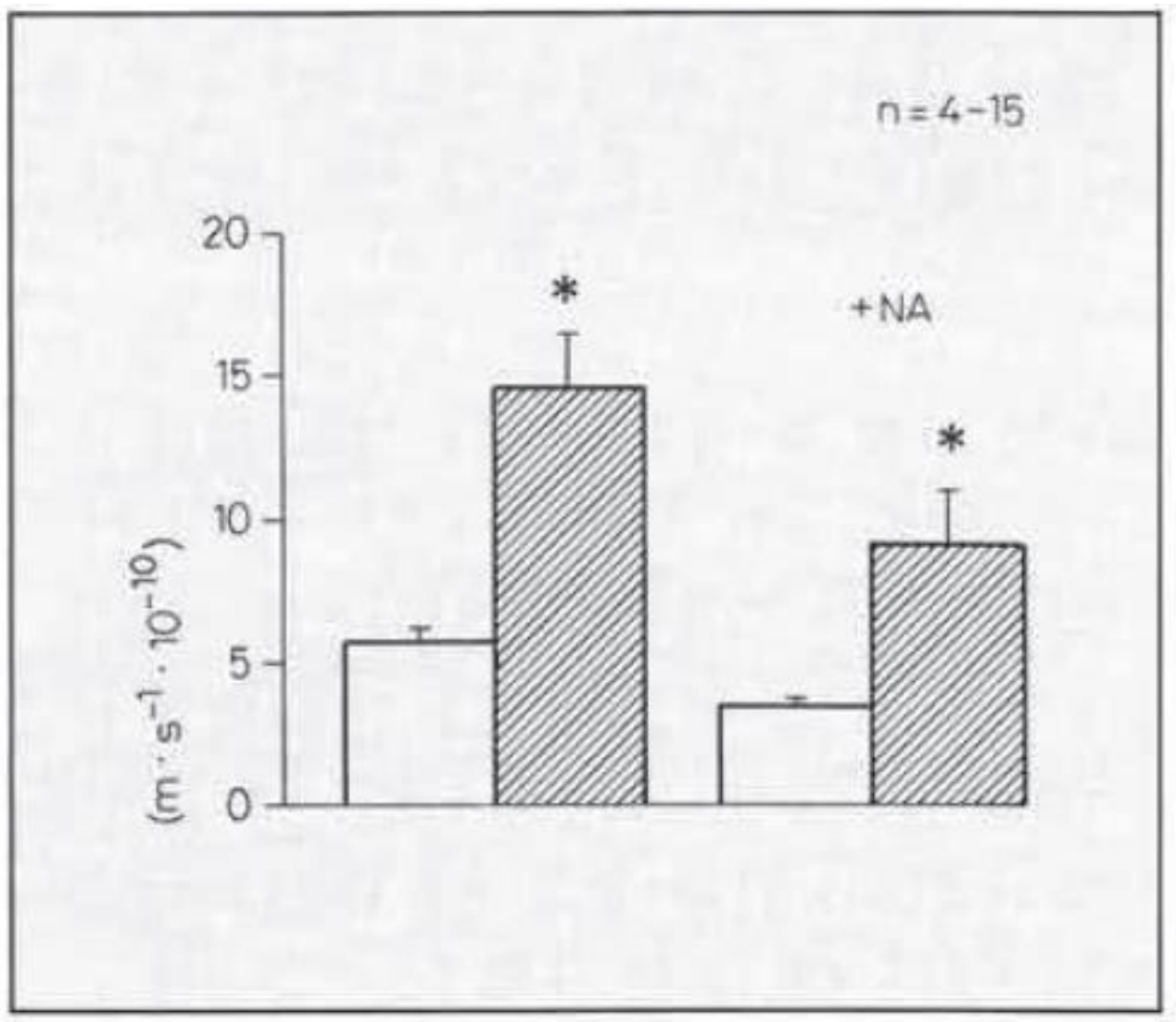

Fig.3. Effect of pinacidil ( $10 \mu \mathrm{M}$, hatched bars) on $\mathrm{K}^{+}$permeability of rat mesenteric small arteries either when relaxed (left) or when activated (right) with noradrenaline $(10 \mu \mathrm{M})$, compared to control (open bars). Bars show SEM [from data in 7]. 


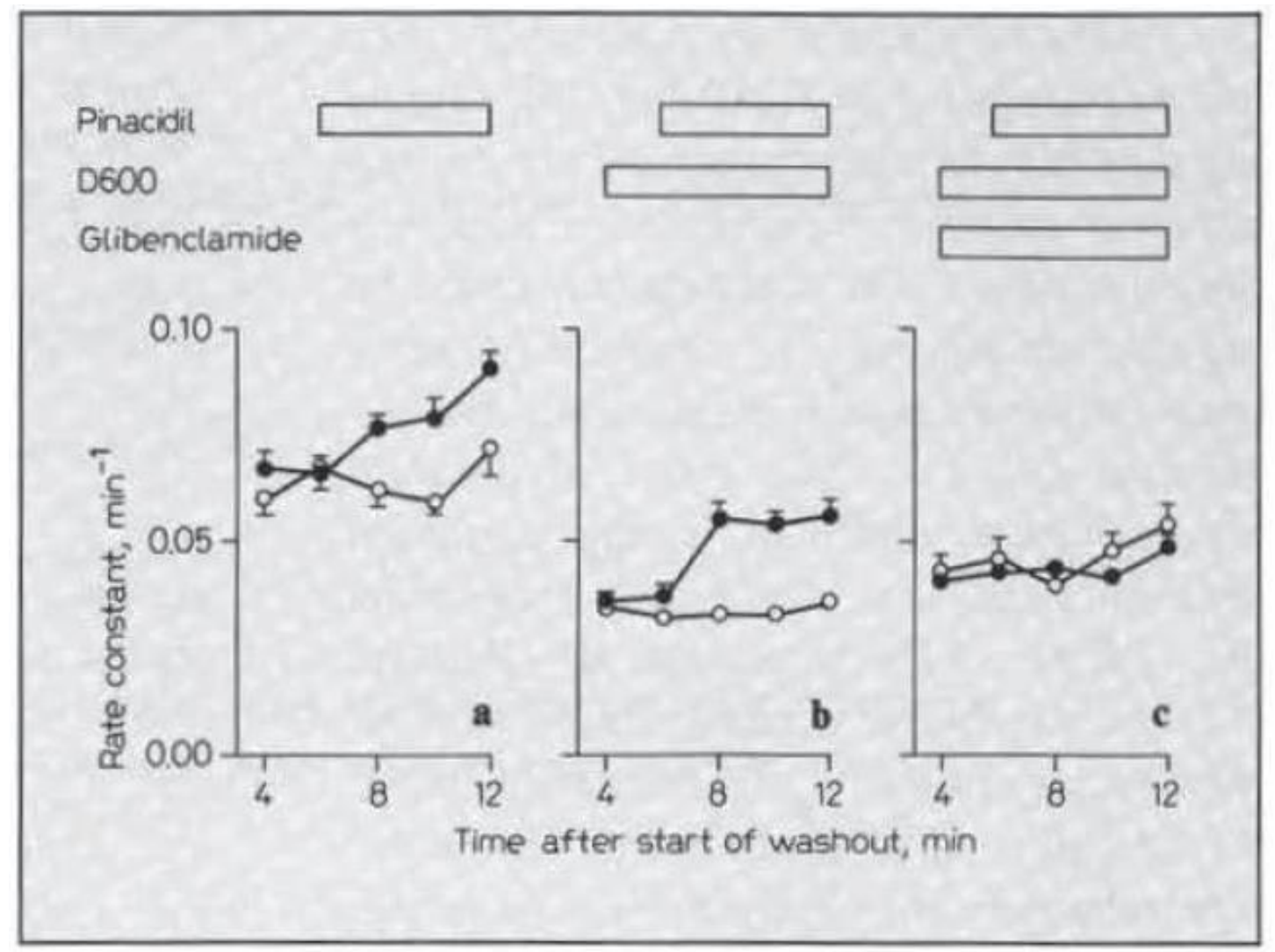

Fig.4. Effect of pinacidil $(10 \mu \mathrm{M})$ on ${ }^{86} \mathrm{Rb}$ efflux rate constants from rat mesenteric small arteries. Vessels loaded with ${ }^{86} \mathrm{Rb}$ as described in Videbaek et al. [7], and washed out in high potassium solutions $\left(\left[\mathrm{K}^{+}=125 \mathrm{mM}\right)\right.$ containing noradrenaline $(\mathrm{NA})$, pinacidil and D600, as indicated. Each point shows mean \pm SEM for $15-16$ vessels. a Enhancement of ${ }^{86} \mathrm{Rb}$ efflux by pinacidil. b Failure of D600 $(1 \mu \mathrm{M})$ to inhibit the effect of pinacidil. c Glibenclamide $(10 \mu \mathrm{M})$ inhibition of the effect of pinacidil. 